\title{
$1 \quad$ Mapping the landscape of chromatin dynamics during naïve CD4+ T-cell
}

3 Authors: Muhammad Munir Iqbal ${ }^{1}$, Michael Serralha ${ }^{1}$, Parwinder Kaur ${ }^{2}$, David Martino ${ }^{1,2,3, *}$.

\section{Affiliations:}

$6{ }^{1}$ Telethon Kids Institute, Northern Entrance, Perth Children's Hospital, 15 Hospital Avenue, Nedlands,

7 Western Australia, 6009, Australia

$8{ }^{2}$ Faculty of Science, UWA School of Agriculture and Environment, The University of Western Australia,

935 Stirling Highway, Nedlands, Western Australia, 6009, Australia

$10{ }^{3}$ Centre for Food and Allergy Research, Murdoch Children's Research Institute, University of Melbourne, 11 Flemington Road, Parkville, Victoria, 3053, Australia

12

13 *To whom correspondence should be addressed: David.Martino@telethonkids.org.au, Telethon Kids

14 Institute, Perth Children's Hospital, 15 Hospital Avenue, Nedlands, Perth, WA, 6009.

15

16 Running title: Chromatic Dynamics of T-cell Activation

17

18 Key Words; T-cell, chromatin 


\section{Abstract:}

20 T-cell activation induces context-specific gene expression programs that promote energy generation and

21 biosynthesis, progression through the cell cycle and ultimately cell differentiation. The aim of this study

22 was to apply the omni ATAC-seq method to characterize the landscape of chromatin changes induced by

23 T-cell activation in mature naïve CD4+ T-cells. Using a well-established ex vivo protocol of canonical T-

24 cell receptor signaling, we generated genome-wide chromatin maps of naïve T-cells from pediatric donors

25 in quiescent or recently activated states. We identified thousands of individual chromatin accessibility peaks

26 that are associated with T-cell activation. The majority of these were localized to intronic and intergenic

27 enhancer regions, marked by active histone modifications whilst quiescence was maintained by repressive

28 histone marks. Regions of activation-associated gains in chromatin accessibility were enriched for well-

29 known pioneer transcription factor motifs, and super-enhancer regions associated with distinct gene

30 regulatory networks. These cis-regulatory elements together brought about distinct transcriptional

31 signatures in activated cells including TNFa-NFkB signaling, hormone-responsive genes, inflammatory

32 response genes and IL2-STAT5 signaling. Our data provides novel insights into the chromatin dynamics

33 and motif usage of T-cell receptor signaling events in early life. The characterized pathways demonstrate

34 the utility of chromatin profiling techniques applied to bio-banked samples for characterizing gene

35 regulatory elements. 


\section{Introduction}

Naïve CD4+ T-cells circulate through the periphery in an actively maintained state of quiescence, ready to mount a robust immune response to pathogens. Quiescent T-cells maintain a tightly condensed chromatin architecture (Rawlings et al. 2010) and cellular program of low energy expenditure whilst surveying for cognate antigen (Wolf et al. 2020), and rapidly undergo substantial re-programming following activation, transitioning toward highly proliferative effector cells. Activation of naïve T-cells initiates rapid functional adaptations which, over the course of days, evolves into heterogenous effector

43 fates with unique helper and regulatory functions with the potential for establishing long-lived memory phenotypes. Activated T-cells rapidly increase nutrient uptake, ramp up translational activity and switch to glycolytic pathways to provide the energy required to support cell growth(Phan et al. 2017), a massive proliferative response and the acquisition of effector functions. These adaptive changes are well understood to be underpinned by epigenetic (Tough et al. 2020), metabolic (Phan et al. 2017), transcriptional and proteomic (Wolf et al. 2020) changes.

At the nuclear level, T-cell receptor (TCR) signaling induces dynamic re-positioning of nucleosomes at promoters and enhancers to allow for transcriptional changes (Schones et al. 2008). These dynamic changes in the chromatin landscape enable interactions between sequence-specific transcription factors $(\mathrm{TF})$ with regulatory DNA elements. Although promoters are the primary sites of transcription initiation, enhancers are major determinants of cell-specific transcriptional and physiological adaptations (Heinz et al. 2015). The assay for transposase-accessible chromatin (ATAC-seq) has gained in popularity as a method to map chromatin accessibility corresponding to TF binding sites and nucleosome positioning

56 (Schep et al. 2015) at the genome-wide scale, due to its high resolution and low cell input, enabling ex

57 vivo analyses (Buenrostro et al. 2013; Scharer et al. 2016). A more recent variant of the ATAC-seq method known as omni-ATAC has demonstrated advantages for removing unwanted mitochondrial reads

59 and exhibits better performance on fixed and flash-frozen material (Corces et al. 2017). ATAC-seq

60 integrated with TF binding motifs has proven increasingly useful for uncovering the dynamic changes in 61 enhancer landscapes and predicting key regulatory events that bring about chromatin remodeling. The 
62 dynamic remodeling of enhancer landscapes and differential TF motif usage is a characteristic of distinct

63 T-helper subsets (Bonelli et al. 2014). The majority of data available to date has been performed on

64 neonates (Henriksson et al. 2019), adults (Yukawa et al. 2020; Wolf et al. 2020) or murine cells

65 (Rawlings et al. 2010; Chisolm et al. 2017; Champhekar et al. 2015; Ungerbäck et al. 2018), and

66 there is a paucity of data on infants and young children. Thus, our goal was to examine the utility of omni-

67 ATAC for characterizing chromatin dynamics and inferring gene-regulatory networks in paediatric bio-

68 banked samples. We have previously described deficiencies in T-cell activation transcriptional networks

69 and activation-induced regulation of DNA methylation in young infants who developed IgE-mediated food

70 allergy (Martino et al. 2011, 2018). Studying T-cell activation responses at the molecular level has

71 translational potential for understanding disease mechanisms and uncovering novel molecular targets.

In this study we isolated mature naïve T-cells from 6 healthy paediatric donors and studied

73 chromatin dynamics in the canonical T-cell receptor signaling pathway using an identical protocol as

74 published previously by us (Martino et al. 2018). This allowed us to analyze stimulation-dependent

75 chromatin changes in the context of previously collected transcriptomic data. By integrating additional

76 epigenetic data sets we undertook an epigenomic analysis of the paediatric T-cell activation response. Our

77 data are largely consistent with previous studies, demonstrating the utility of omni-ATAC for characterizing

78 the enhancer landscape and motif usage in paediatric bio-banked samples, as a prelude to future studies of

79 disease mechanism.

80 Results

81 Post-alignment $Q C$

82 We isolated mature naïve T-cells from 6 healthy infants and studied chromatin dynamics in the

83 canonical T-cell receptor signaling pathway using an identical protocol as published previously by us

84 (Martino et al. 2018). Naïve T-cells were activated with anti-CD3/anti-CD28 beads for 72 hours (activated

85 nCD4T) with matched un-stimulated control condition (quiescent nCD4T). Using cell tracing dyes this

86 protocol results in $3-4$ distinct T-cell divisions expanding the clonal population on average by 2 -fold

87 (expansion index 2.044 [range 1.80 - 2.23], Fig. 1A). After 72 hours all cells were recovered for chromatin 
profiling. Previous analysis demonstrated activated cells harvested in this phase represent a transitional population of highly proliferative early effector phenotypes (Martino et al. 2018). We generated maps of genome-wide chromatin accessibility to identify epigenomic elements that bring about the stimulation

91 response in resting and activated cells. Post-alignment quality control indicated high mapping efficiency

92 with overall alignment rates $96 \%$ or higher. Most reads were enriched at transcriptional start sites for both

93 activated and resting nCD4T. Fragment length distribution plots yielded high resolution of nucleosome-

94 free and nucleosome-occupied reads. Reads were highly enriched at universal DNAse1 hypersensitivity

95 regions identified by the ENCODE consortium (Yue et al. 2014) and enhancer regions indicative of

96 regulatory DNA elements. Activated nCD4T cells exhibited a higher number of reads at promoter regions

97 compared with resting nCD4T (Supplemental Fig. 1).

A nCD4T Proliferation B

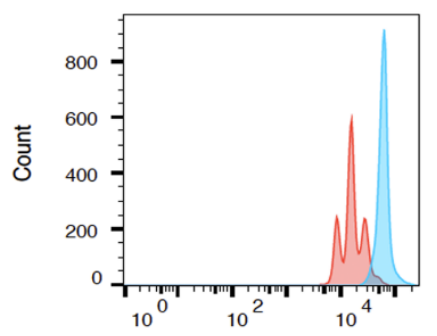

C

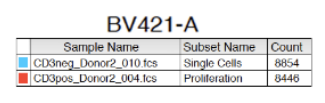

D

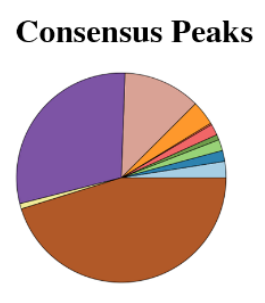

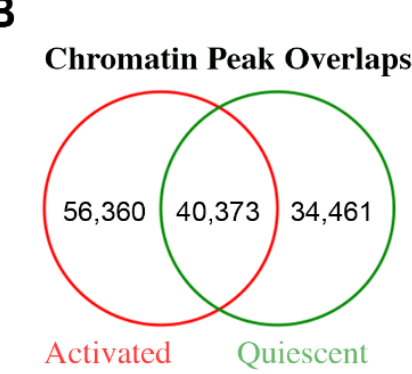
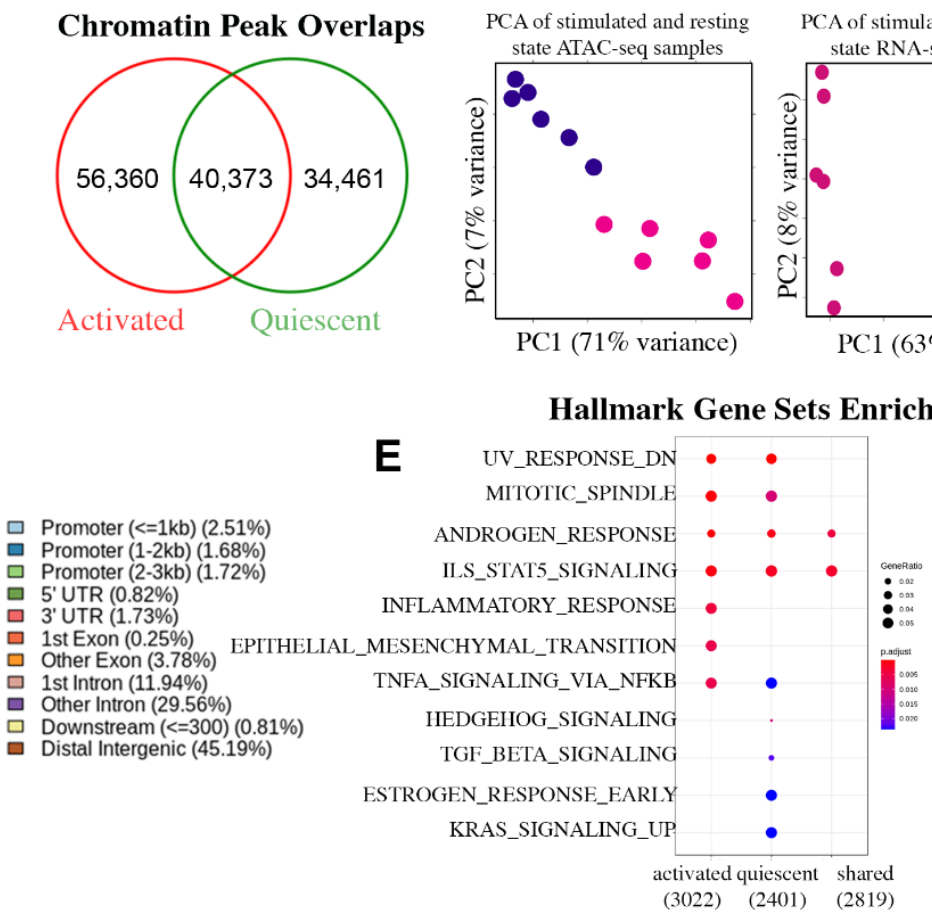

PC1 (71\% variance)

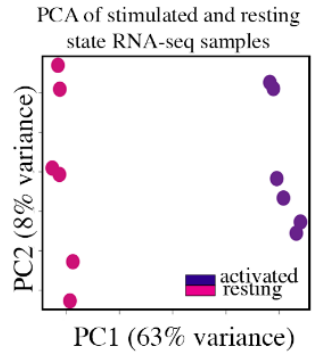

PC1 (63\% variance)

Hallmark Gene Sets Enrichment 
102 counts of chromatin accessibility peaks. (C) Principal component analysis of ATAC-seq peaks (left) and

103 RNA-seq transcripts (right). Each sample is projected into 2D space in a way that best explains variance.

104 (D) Annotation of consensus chromatin accessibility peaks to genomic regions of the hg19 genome. (E)

105 Gene sets enrichment analysis of molecular signatures enriched in accessibility peaks.

\section{Open chromatin peak occupancy}

Compared to resting nCD4T, the genomes of activated cells were more accessible as evidenced by

109 a larger number of open chromatin peaks. We identified 74,834 consensus peaks in quiescent nCD4T and

11096,733 peaks in activated nCD4T, with 40,373 common peaks (Fig. 1B). Principal component analysis of

111 chromatin profiles and publicly available RNA-seq data from our previous study (GSE114064) revealed

112 distinct chromatin signatures for activated and quiescent nCD4T, concomitant with distinct transcriptional

113 programs (Fig. 1C). We annotated consensus peaks to the hg19 reference genome and examined the

114 distribution of peaks across genomic features. The pie chart in Fig. 1D indicated that at least two-thirds of

115 peaks were annotated to enhancer regions (distal intergenic and intronic), with only a small percentage of

116 peaks localized to promoter regions. The latter is consistent with the typical pattern of ATAC peaks

117 representing a mixture of different cis-regulatory elements such as enhancers and promotors (Thurman et

118 al. 2012). Hypergeometric testing of peaks revealed quiescent and activated nCD4T shared many regions

119 of open chromatin at genes in the IL2-STAT5 signaling pathway, TNFa-NFkB signal transduction genes

120 and hormone response genes (Fig. 1E). Peaks of chromatin accessibility unique to activated cells were

121 enriched at inflammatory response genes and genes involved in the gain of migratory capacity represented

122 by the 'epithelial-mesenchymal transition' pathway. In contrast, peaks of accessibility that were unique to

123 resting nCD4T were enriched in TGF-Beta signaling, estrogen response genes and KRAS signaling (Fig.

124 1E).

\section{Differential Binding Analysis}

126 We quantified the number of differentially accessible activation-induced chromatin changes by 127 formally testing MACS2 peaks between quiescent and activated nCD4T. Short-term activation of the T- 
128 cell receptor induced substantial changes in the chromatin landscape comprising 43,269 chromatin peaks $129(\mathrm{q}<5 \%)$ that were differentially accessible, of which 5,607 exhibited a minimum absolute log2 fold change 130 in accessibility of $+/-2.0$ (Fig. 2A). Of the 5,607 differentially accessible peaks, a total of 1,089 peaks gained 131 accessibility in activated nCD4T whilst 4,518 peaks reduced in accessibility (Fig. 2B). Chromatin regions 132 that gained accessibility significantly (FDR < 0.05) coincided with active chromatin marks $(\mathrm{H} 3 \mathrm{~K} 4 \mathrm{me} 1$, $133 \mathrm{H} 3 \mathrm{~K} 27 \mathrm{ac}$ ) in primary T-cells, whilst peaks that reduced in accessibility upon activation coincided with 134 repressive marks (H3K27me3 see refs in Wong) (Fig. 2B). We identified putative transcription factor 135 binding sites within regulatory regions that gained accessibility. In total 114 transcription factor motifs were 136 enriched (Fisher's exact $\mathrm{P}<0.05$ ) within regions that gained accessibility (Fig. $2 \mathrm{C}$ ). The most enriched motif 137 was SPIB, a transcriptional activator that acts as a lymphoid specific enhancer to promote the development 138 of IFN-y producing cells ( $\mathrm{Li}$ et al. 2014). In addition to this novel finding, we identified several well139 known factors with a previously described role in TCR signaling and differentiation (STAT5b, JUN, FOS, 140 BACH1, NFATC4, BATF3). This analysis suggested that chromatin landscape changes associated with $141 \mathrm{nCD} 4 \mathrm{~T}$ activation prime for differentiation into T-effectors. In support of this, we found that regions of 142 accessible chromatin significantly coincided with DNAseI hotspots found in differentiated T-effectors 143 (Th17, Th1, Th2, Tregs) and were relatively depleted in hotspots unique to naïve precursors and monocytes 144 (as a negative control (Fig. S1)). To corroborate the motif prediction analysis, we used transcription-factor 145 ChIP-seq data available through the ENCODE consortium to test whether regions of that gain accessibility 146 in response to activation coincide with experimentally derived ChIP-seq transcription factor peaks. There 147 were 7 transcription factor ChIP-seq datasets in ENCODE from lymphoblastoid cells lines available for 148 testing (BATF, BHLH, CTCF, JUND, POUF, STAT5A, TCF3), and enrichment analysis indicated all 7 149 transcription factors signal peaks significantly (Adjusted $\mathrm{P}<0.05$ ) coincided with ATAC peaks of 150 accessibility more than expected by chance (Fig. 2D). JUND and STAT5 exhibited the greatest overlap 151 with ATAC accessibility peaks. As expected, when we performed the same enrichment testing on ATAC 152 peaks that lost accessibility, there was no evidence of enrichment (Adjusted P $>0.05$, data not shown). We 153 next input the list of 114 identified transcription factors into the GSEA molecular signatures database and 154 performed gene set enrichment analysis, which revealed strong enrichment for the TNFa-NFkB signal 
A

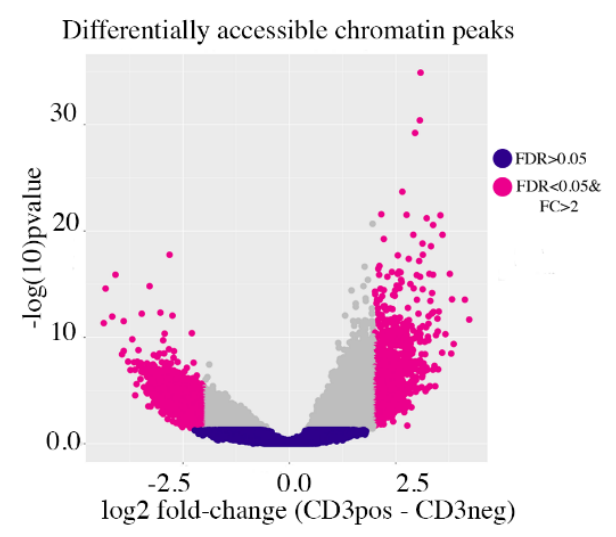

C

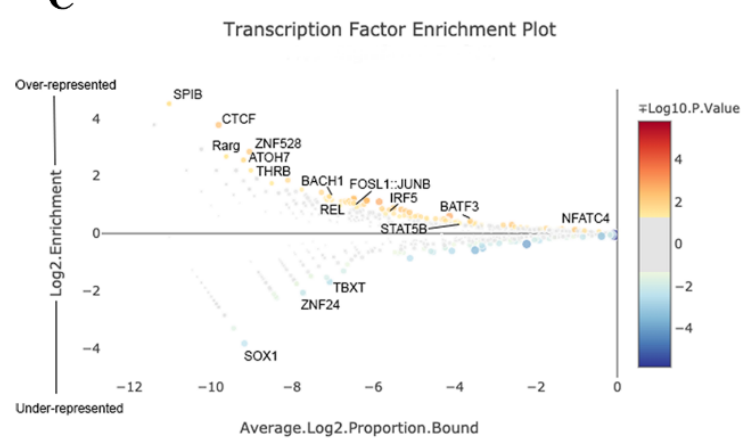

B

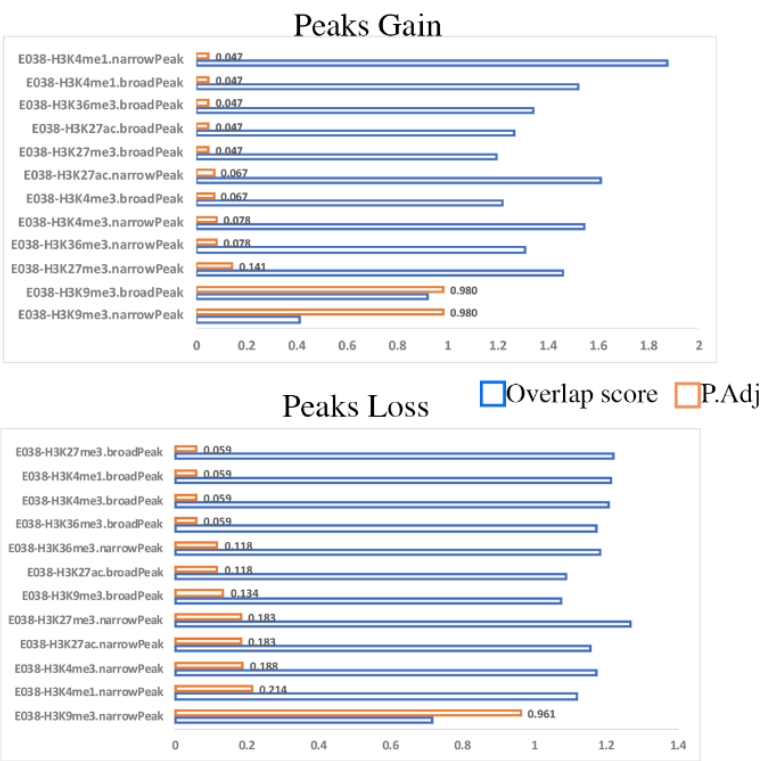

D

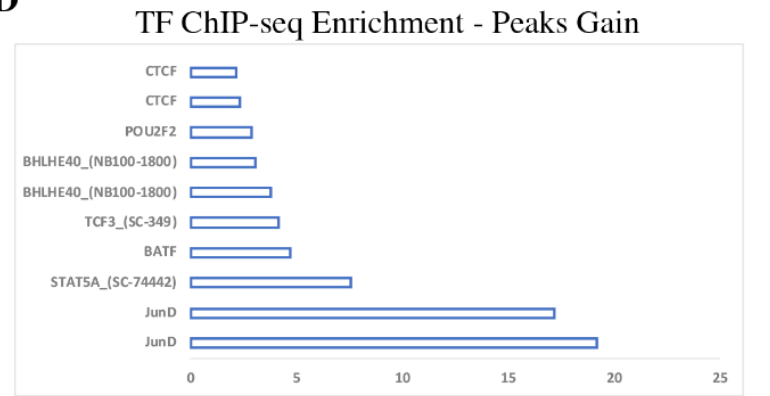

Figure 2 - Differential accessibility of chromatin peaks in activated versus resting cells. (A) Volcano

160 plot of differentially accessible peaks. Each data point represents a consensus peak. (B) Statistical overlap

161 of stimulus-dependent accessible regions with histone ChIP-seq peaks from primary T-cells. (C) Enriched stimulus-dependent accessible regions with transcription factor ChIP-seq peaks from ENCODE. 
167 Super-enhancers drive T-lymphoid specific gene regulatory networks

168 Recent studies suggest core transcription factors can bind clusters of enhancer elements, known as

169 super-enhancers, that can drive interconnected gene regulatory networks (Hnisz et al. 2013). We used the

170 SEanalysis tool to query regions of chromatin accessibility gains against $>330 \mathrm{k}$ super-enhancers catalogued

171 from broad H3K27Ac peaks identified from ENCODE CD4+ T-cells. Activation-induced regions that

172 gained chromatin accessibility overlapped 16 known CD4+ T-cell super-enhancers (Table S2).

173 Visualization of ATAC-peaks mapping to the regions identified by the SEanalysis tool showed clear

174 evidence of accessibility gains across the broad region in response to stimulation with concomitant changes

175 in gene expression of nearby transcripts (Fig. 3A and B). These super-enhancer elements formed part of a

176 larger complex gene regulatory network. For example, a super-enhancer annotated to $A R I D 4 B$, a subunit of

177 a co-repressor complex, contains 9 transcription factor binding motifs that were significantly enriched

178 (hypergeometric $\mathrm{FDR}=2 \times 10^{-08}$ ) in interferon alpha/beta signaling, JAK-STAT signaling, interleukin

179 signaling and other pathways (Fig. 3C). 
A

ARID4B ATAC peaks

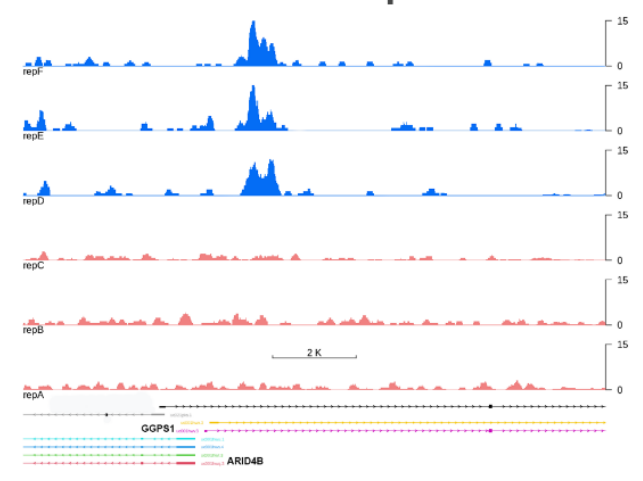

C

ARID4B Super-Enhancer Network

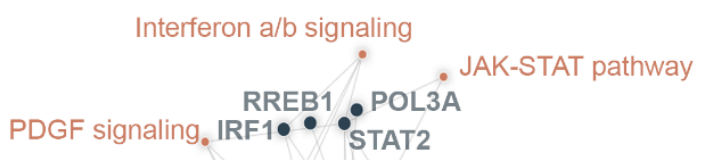

B
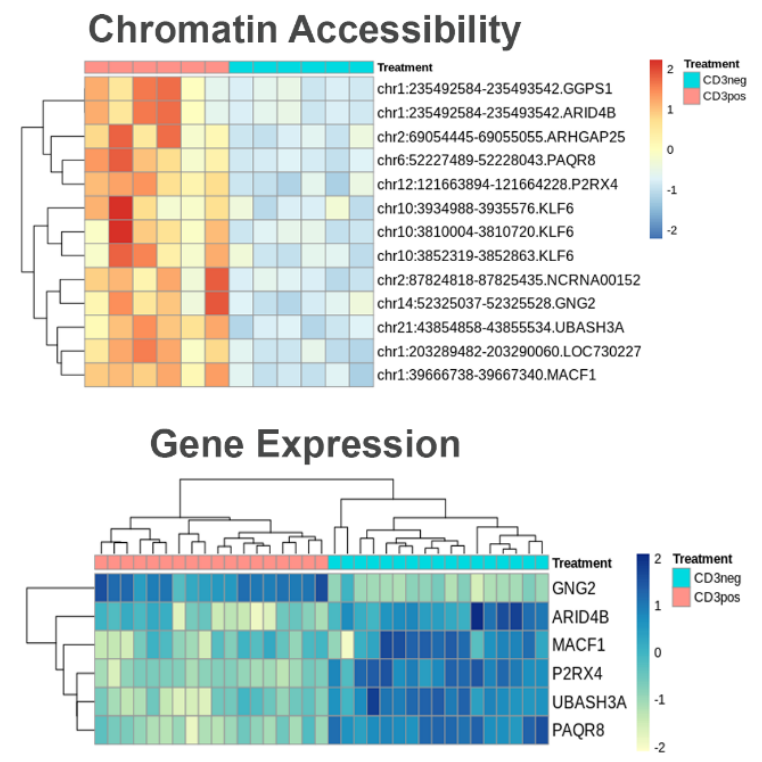

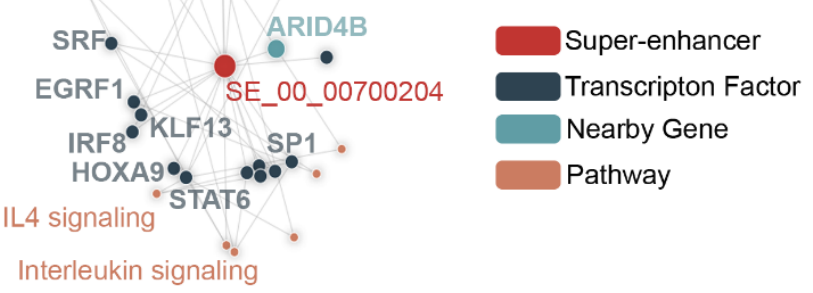

181 Figure 3 - Super enhancer regions detected in stimulus-dependent accessible regions. (A) Signal

182 track of ATAC-seq peaks in activated (blue) and quiescent (red) nCD4T. RefSeq transcripts are show in

183 the track below. (B) Heatmap of ATAC-seq peaks and RNA-seq transcripts. Rows represent peaks or

184 transcripts and columns represent samples. Cells are colored according to z-score. (C) ARID4B super-

185 enhancer network diagram showing the regulatory relationship between the identified super-enhancer 186 peak (SE_00_00700204), transcription factor motifs enriched at this peak, and their over-represented

187 down-stream pathways.

\section{Relationship to transcriptional changes}

We next sought to determine how differentially accessible regions associated with activation are

191 related to changes in gene expression. Using transcriptomic data from our previous naïve CD4T study with

192 harmonized laboratory stimulation protocol (GSE114064), we filtered the data set to transcripts overlapping 
193 differentially accessible peaks and plotted mean gene expression profiles in activated and quiescent nCD4T.

194 We found that average gene expression levels for transcripts located within differentially accessible regions

195 were broadly similar across treatment conditions (Fig. 4A). Given the high level of enrichment of ATAC-

196 seq peaks in enhancer elements rather than promoters (Fig. 1D), it stood to reason that chromatin changes

197 would likely have more subtle cis- regulatory effects on gene expression that would not be obvious as a

198 simple $1: 1$ relationship. To explore this further, we first performed a gene sets enrichment analysis on

199 differentially accessible ATAC peaks. Stimulus-responsive regions that gained accessibility were enriched

200 at INFLAMMATORY_RESPONSE and genes in the IL2-STAT5 pathway, whereas those that lost

201 accessibility were enriched in UV RESPONSE, HEDGEHOG and KRAS signaling genes (Fig. 4B). We

202 then tested the hypothesis that expression of these pathways as a whole would differ across treatment

203 conditions. We reasoned that IL2-STAT5 and INFLAMMATORY_RESPONSE signatures would be

204 enriched in activated nCD4T compared with resting $\mathrm{nCD} 4 \mathrm{~T}$ given the activation-induced gain in

205 accessibility of genes in these pathways. Consistent with this we found strong enrichment (adj. P=0.02 IL2

$206 \&$ adj.P=0.04 INFLAMMATORY) for these molecular signatures in activated nCD4T (Fig. 4C), but there

207 was no evidence of enrichment in resting nCD4T (adj. P = 0.3, IL2 \& INFLAMMATORY). Likewise, we

208 found evidence of enrichment transcripts in UV response genes among quiescent nCD4T only but not

209 activated nCD4 T (adj. P=0.03, Fig. 4C). 
A

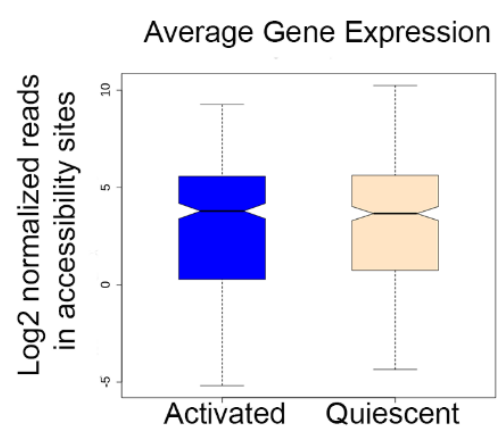

B

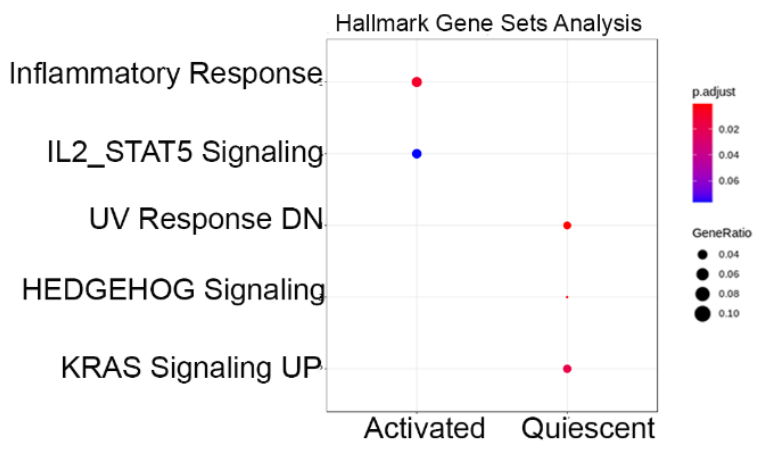

C

GSEA: nCD4T activated

GSEA: nCD4T quiescent
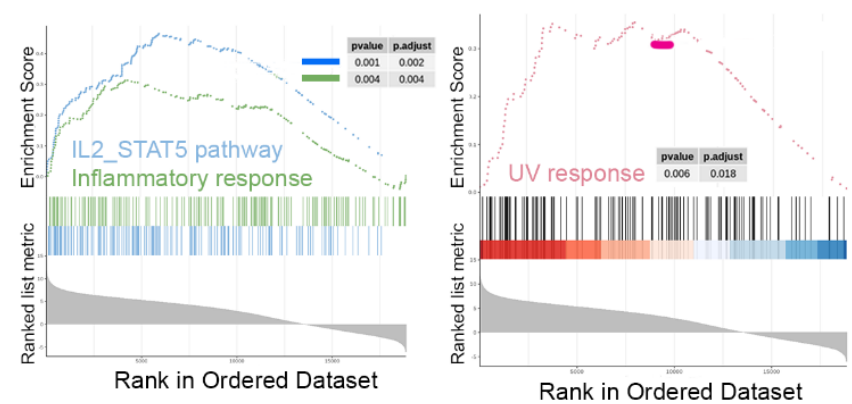

211 Figure 4 - Functional analysis of gene expression at stimulus-dependent accessible regions. (A)

212 Boxplot of expressed genes in accessibility sites shows no evidence of global changes in transcriptional

213 output. (B) Predicted enriched molecular signatures at differentially accessible peaks (FDR $<0.05$ and

$214 \log 2 \mathrm{FC}+/-2)$. (C) GenesSets enrichment scores of expressed transcripts validating predicted molecular

215 signatures from ATAC-seq peaks. Top panels show overall enrichment score for the pathway tested. The

216 middle panel tick marks show where the members of the gene set appear in the ranked list of genes. The

217 bottom panel shows the value of the gene's correlation with phenotype.

219 Collectively, this analysis suggests that stimulus-dependent chromatin changes drive a broader gene 220 regulatory network comprising both direct and indirect interactions. To illustrate the latter point we 221 identified differentially expressed genes by comparing the transcriptomes of quiescent versus activated 222 nCD4T. In total, 3876 genes were differentially expressed (FDR $<0.05 \& \log \mathrm{FC}>2$ ). We performed 223 ontology enrichment analysis on the list of differentially expressed genes (DEGs) and differentially 
accessible regions (DARs). The circos plot in Fig. 5A show the direct overlap between the list of DEGs and

A

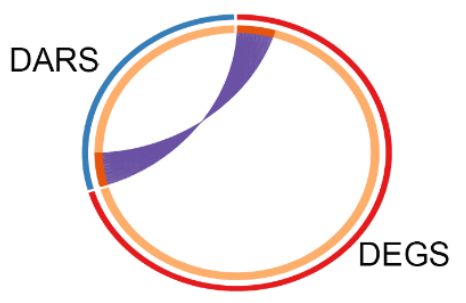

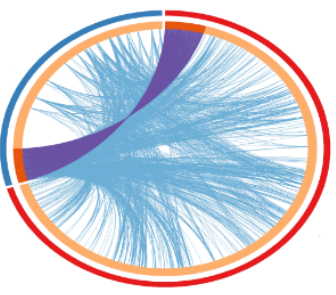

B

Figure 5 - Direct and indirect relationships between stimulus-responsive chromatin peaks and genes.

(A) Circos plot of differentially accessible regions (DARS: FDR $<0.05 \& \log 2 \mathrm{FC}+/-2$ ) and differentially expressed genes (DEGS: FDR $<0.05 \& \log 2 \mathrm{C}+/-2$ ). Each gene in both lists is represented on the inner arc. Dark orange colour joined by purple chord represents genes that appear in both lists and light orange colour are unique genes. Blue lines link the different genes where they fall into the same statistically enriched ontology term. (B) Statistically enriched terms (GO/KEGG terms, canonical pathways, etc.) in chromatin 238 regions and differentially expressed genes. The heatmap cells are colored by their p-values, white cells 239 indicate the lack of enrichment for that term in the corresponding gene list.

\section{Discussion}

242 T-cell activation induces global remodeling of chromatin accessibility in an orderly and timely manner.

243 These epigenetic changes are coincidental with specific gene regulatory networks that bring about changes 
244 in cellular metabolism, proliferative capacity and effector function(Bonelli et al. 2014). In this study we 245 compared genome-wide chromatin accessibility maps between quiescent and activated naïve CD4+ T-cells.

246 Consistent with previous studies (Rawlings et al. 2010) we found that TCR signaling induces wide-spread 247 de-condensation of chromatin, as evidence by substantially higher $(22,000)$ open chromatin peaks detected

248 in activated cells. The transition to proliferative early effectors appears highly dependent on chromatin 249 remodeling and less so for other epigenetic changes. Our previous genome-wide studies of DNA 250 methylation dynamics in CD4+ T-cell activation revealed no substantial changes in methylation dynamics 251 at 48 hours post-activation (Martino et al. 2012) and $558 \mathrm{CpG}$ dinucleotides that were stimulus-responsive 252 at 72 hours(Martino et al. 2018). This is in contrast to the 43,000 chromatin accessibility changes we 253 detected at the genome-wide level, around 5,000 of which exhibited very large changes. Similarly, 254 Rawlings et al also reported that TCR-induced nuclear de-condensation was not dependent on CpG 255 methylation, nor any substantial net changes in global histone modifications (Rawlings et al. 2010). 256 Chromatin remodeling is therefore a highly dynamic epigenetic mediator of the T-cell activation response. Chromatin accessibility modulates DNA interactions with transcription factors and the transcriptional machinery. Stimulation associated regions that gained accessibility in our data set were 259 marked with active histone modifications, whilst regions that condense overlapped repressive histone 260 modifications that may serve to suppress alternative cell fates and lineages. In our data set we identified 261 strong enrichment for pioneering transcription factor motifs SPI-1 (also known as PU.1), CTCF and BATF 262 in regions that gain accessibility. PU.1 is a well-established pioneer factor in early T-lineage commitment 263 that binds gene enhancers (Ungerbäck et al. 2018), supports proliferation and restrains alternative 264 lineages (Champhekar et al. 2015). Binding of SPI-1 can induce chromatin opening and maintain 265 accessibility at target sites. The CTCF DNA binding zinc finger transcription factor plays a spatially 266 organizing role in the genome and promotes precise regulation of developmental gene expression programs. 267 In CD4-T cells, changes in CTCF binding patters are associated with interleukin-2 sensitive metabolic 268 changes (Chisolm et al. 2017). The recruitment of CTCF in T-cells is known to be BATF-dependent, and 269 we detected enrichment for BATF motifs in regions that gain accessibility on activation (Phan et al. 2017). 
270 We also found enrichment of AP-1 family binding motifs (Fos, Jun) which are known pioneer factors that

271 are dramatically up-regulated in response to T-cell activation (Yukawa et al. 2020). Both AP-1 and NFAT

272 (detected in our dataset) are known to play a role in super-enhancer formation in response to T-cell

273 activation (Yukawa et al. 2020). Our analysis identified 13 super-enhancers that were associated with

274 transcriptional changes. Collectively these data characterize the motif usage that bring about the activity

275 change into early effector 'Th0' progeny cells. It also highlights the power of the omni-ATAC technique

276 for identifying key regulatory proteins for experimental follow-up with specific transcription-factor ChIP.

277 The relationship between chromatin landscape dynamics and transcriptional state changes was

278 complex in our interpretation. We found that stimulus-dependent chromatin accessibility changes were

279 enriched in IL2-STAT5 signaling and inflammatory response genes, and these molecular signatures were

280 significantly enriched in activated cells. These are extremely well characterized transcriptional pathways in

281 T-lymphocyte responses (Ross and Cantrell 2018), thus validating the utility of the omni-ATAC

282 technique for deciphering the underlying gene regulatory networks associated with chromatin state changes.

283 It is noteworthy that we did not identify obvious direct relationships between chromatin changes and gene

284 expression, which may be expected given the majority of chromatin remodeling occurred at intronic or

285 distal intergenic sites, suggesting more complex regulation of gene expression. A recent proteomic study

286 has demonstrated that naïve T-cells maintain a reservoir of glycolytic enzymes and un-translated mRNAs

287 that are immediately mobilized in response to activation, allowing naïve cells to kick-start glycolysis and

288 protein synthesis (Wolf et al. 2020). Thus, chromatin responses account for only a portion of the T-cell

289 activation transcriptional response and comprise one of several regulatory mechanisms that underpin T-cell

290 responses. An alternative explanation and potential limitation are that directly relationships were difficult

291 to infer as the gene expression data were from another experiment and therefore may have captured the

292 same biological snapshot albeit at a slightly different time. Although the ex vivo protocols were

293 harmonized, we cannot rule out experimental variation as posing challenges for inferring direct

294 relationships. 
The strengths of this study include utilizing a strategy to study canonical TCR signaling by presorting naïve CD4+ T-cells, resulting in a pure and homogenous population. This circumvents difficulties

297 in interpretation posed by co-culture with antigen-presenting cells as chromatin dynamics are likely 298 influenced by secreted factors and cell to cell interactions from accessory cells. By focusing on naïve CD4+ 299 cells rather than total CD4+ cells, which are a mixed population, we avoided confounding due to cellular 300 heterogeneity. We utilized existing RNA-seq data and integrating ChIP-seq and DNAse1-sep datasets from 301 the ENCODE project. These integrations mutually validate the reliability of the ATAC-seq data and aid in

302 biological interpretation (Yan et al. 2020). Caveats include studying only one time-point in the T-cell 303 activation response, and lack of functional data on cytokine responses and cell surface marker changes.

304 This was deemed outside the scope of the current study, as we have characterized these responses in 305 previous studies (Martino et al. 2018)and our focus in the current study was on the chromatin response.

306 We also identified areas of improvement in the omni-ATAC protocol, namely reducing the number of PCR 307 cycles to reduce duplication rates. We were not able to perform a motif foot printing analysis as we did not 308 have sufficient depth of sequencing to accurately call footprints. Overall, this study characterized the 309 chromatin dynamics that bring about the 'Th0' early effector progeny and their respective transcriptional 310 state. Importantly, we have done this ex vivo on infant biospecimens demonstrating an approach amenable 311 to paediatric cohort studies. Our future studies will build on the methodology here to study the epigenetic 312 regulation of T-cell activation in disease phenotypes such as allergy and autoimmunity.

\section{Materials and Methods}

\section{Subject selection}

316 Subjects were recruited through Princess Margaret Hospital in Perth, Western Australia as part of a 317 community-based program of allergy prevention. All subjects used in this study underwent prospective 318 clinical assessments at 1,2.5 and 5 years of age, including phenotyping for allergic outcomes and general 319 health and donated venous blood for cryopreservation according to institutional ethics committees. 320 Inclusion criteria for selecting biospecimens for this study included equal numbers of males $(\mathrm{n}=3)$ and 
321 females $(n=3)$, subjects were 1-year of age at time of biospecimen collection, subjects did not receive any

322 interventions, subjects had more than 1 vial of cryopreserved peripheral blood mononuclear cells (PBMC)

323 in the biobank. Exclusion criteria included any congenital malformations, any primary immune deficiency

324 or clinically significant illness that would affect normal hematopoietic development. General characteristics

325 of the cohort are provided in Table S3.

\section{Isolation, activation and expansion of naïve CD4+ T-cells}

Cryopreserved PBMC were thawed in RPMI media (Gibco) supplemented with $10 \%$ fetal bovine serum

(FBS), Pen-Strep and benzonase $(25 \mathrm{U} / \mathrm{mL})$ maintained in a 37-degree water bath. After thawing, cells were

washed twice, counted and viability checked by trypan blue. Cell recoveries ranged from $8-20$ million

PBMC with viabilities higher than 90\%. Naïve CD4+ T-cells (CD3+CD4+CD45RA+CD45RO-) were purified from PBMC using the EasySep Human CD4+ T-cell Isolation Kit (Stemcell Technologies) to $>95 \%$ purity according to manufacturer's instructions. Yield of naïve T-cells ranged from $1-2.5$ million

cells. Naïve CD4+T cells were pre-labelled with 5mM CellTrace Violet division tracking dye (Thermo

335 Fisher) according to manufacturer's instructions and seeded into 96-well polystyrene plates at 80,000 cells

336 per well in RPMI media with 10\% FBS, Pen-Strep and human recombinant interleukin-2 (210U/mL, R\&D

337 systems). For activation, $2 \mathrm{uL}$ of Human T-cell Activator Dynabeads CD3/CD28 (Life Tech) was added to

338 each well reserved for activation, with an equal number of un-activated wells. Cells were incubated for 72

339 hours at 37 degrees and 5\% CO2 before harvesting. At culture end-point, cells were thoroughly

340 resuspended, and beads were removed with replicate wells combined into a single tube for ATAC-seq. A

341 proportion of replicate wells was reserved for proliferation analysis on the BD Fortessa cytometer with

$342405 \mathrm{~nm}$ excitation and 450/40 bandpass emission filter.

\section{Omni ATAC-seq}

345 We employed the omni-ATAC method of Corces. 80,000 viable naïve T-cells were pelleted and lysed in 346 lysis buffer containing 10mM Tris-HCl, $10 \mathrm{mM} \mathrm{NACl,} \mathrm{3mM} \mathrm{MgCL} 2,0.1 \%$ NP40, 0.1\% Tween20 and 
$347 \quad 0.01 \%$ Digitonin for 3 minutes on ice. Cells were washed with $1 \mathrm{~mL}$ of cold wash buffer (lysis buffer without

348 NP40 or Digitonin) and nuclei were pelleted in a centrifuge at 800 RCF for 10 min at 4 degrees. Pelleted

349 nuclei were transposed with Tn5 transposase (Illumina) in TD buffer (Illumina) supplemented with

350 Digitonin $(0.1 \%)$ and Tween20 $(0.01 \%)$ for $30 \mathrm{~min}$ at 37 degrees. Transposed DNA was purified using

351 Zymo DNA Clean and Concentrator-5 Kit (Zymo research) according to manufacturer's instruction. DNA

352 recoveries were measured on the Qubit fluorometer (Invitrogen). Library amplification was performed

353 using Nextera DNA library prep kit with Nextera Index Kit (Illumina) as per manufacturers instruction.

354 The number of PCR amplification cycles was determined by qRT-PCR using Quanitfast SYBR Green PCR

355 mastermix (Qiagen) and Nextera Primer I5 and I7 Indexes for 5 cycles. The number of additional cycles

356 was determined by a second round of qPCR performed on partially amplified libraries based on the CT

357 value reading taken at $1 / 3$ the fluorescence curve. Two step size selection was performed using AMPure

358 XP beads (Beckman Coulter). Libraries were run on the LabChip GXII fragment analyser and quantitated

359 on the Qubit fluorometer. Libraries were shipped on ice to Novogene (China) for pooling and sequencing

360 on 2 lanes of the Illumina HiSeq at 2x150 paired end reads to generate 50 million reads per sample.

\section{Bioinformatics}

363 Raw fastq files were analysed using the Multiqc program to generate QC metrics and were processed using 364 the ENCODE official ATAC-seq pipeline version 1.4 specified here. Briefly, adapters detection and 365 trimming were performed using cutadapt (1.91.) and trimmed reads were aligned using the Bowtie2 (2.2.6)

366 aligner. Mapping statistics were generated with SAMtools (1.7) and SAMstats (0.2.1). Post-alignment

367 filtering of duplicates was performed using Picard (1.126) and bedtools (2.26). Aligned reads were shifted

$368+4 \mathrm{bp}$ for the forward strand and $-5 \mathrm{bp}$ for the reverse strand. Fragment length statistics were generated

369 using Picard (1.126). Peak calling was conducted using MACSv2 (2.1.0) and blacklisted regions were

370 filtered using bedtools (2.26). Irreproducibility analysis was performed on pseudoreplicates using

371 phantompeakqualtools (1.2.1) and IDR (2.0.4) on 300K MACS2 peaks using a threshold of 0.05. Reads

372 were annotated to ENCODE regions using python scripts and bedtools (2.26). 


\section{Data analysis}

375 All data analyses were conducted in R version 4.0.2. MAC2 peaks were coerced to a peakset object using

376 Diffbind (2.16). Consensus peaksets were derived for activated and quiescent cells defined by presence in

377 more than half the replicates in each group. Peaks were annotated to the hg19 genome using ChIPseeker

378 (1.24). Enrichment analysis of peaks in hallmark genesets was conducted using the clusterProfiler package

379 (3.16). Normalized read counts for consensus peaks were computed for each sample using Diffbind, and

380 differential accessibility between activated and quiescent T-cells was determined using a matched pairs t-

381 test using the edgeR package (3.30). Peaks were declared differentially accessible at the genome-wide level

382 of false discovery rate adjusted $\mathrm{P}$-value $<0.05$ and those exhibiting a log 2 fold change of $+/-2$ or greater

383 were further analysed. Peak signal tracks were generated using the rtracklayer package (1.48). Motif

384 detection analysis was conducted using the CIIIDER tool (Gearing et al. 2019) using the JASPAR core

385 vertebrates 2020 reference database using default parameters. Detection of super enhancers was performed

386 using the SEanalysis tool (Qian et al. 2019) to query accessibility peaks overlapping $>330 \mathrm{k}$ super

387 enhancers across 542 cells/tissues annotated in the SEdb database, in Genomic Region Annotation mode

388 using 'closet active' gene-SE linking strategy. We restricted the analysis to blood tissue only, and further

389 filtered the results to only primary CD4+ cells. Motif occurrences in constituent enhancers of super

390 enhancers were identified using FIMO (find individual motif occurrences) at p-value threshold of $10^{-7}$ and

391 enriched pathways were identified using hypergeometric testing at a threshold of FDR-adjusted P-value

$392<0.05$. We used the GSuite hyperbrowser program (Simovski et al. 2017) to perform a statistical analysis

393 of over-representation of accessibility peaks with ENCODE datasets. To determine which ENCODE

394 datasets exhibit the strongest similarity to accessibility regions we used the Forbes coefficient to obtain

395 rankings of tracks, and Monte Carlo simulation to provide a statistical assessment of the robustness of the

396 rankings of data tracks, using a null model derived from randomizing the positions of the accessibility

397 regions relative to query tracks. All p-values were adjusted for multiple testing using the Benjamini-

398 Hochberg method. RNAseq data from GSE114064 were downloaded for a subset of age-matched healthy

399 control infants and TMM normalized count data were voom transformed using limma (3.44.3).

400 Differentially expressed genes were declared by comparing transcript expression levels between activated 
401 and quiescent T-cells using a matched-pairs t-test (limma) at the genome-wide level of FDR-adjusted P-

402 value $<0.05$ and $\log 2$ fold change $+/-2$ or greater. Overlaps between differentially accessible regions and

403 genes as well as pathways enrichment analysis were computed using the metascape tool under default

404 settings (Zhou et al. 2019). Statistically enriched terms (GO/KEGG terms, canonical pathways, etc.) in

405 chromatin regions and differentially expressed genes were identified and accumulative hypergeometric p-

406 values and enrichment factors were calculated and used for filtering. Remaining significant terms were

407 then hierarchically clustered into a tree based on Kappa-statistical similarities among their gene

408 memberships. Then 0.3 kappa score was applied as the threshold to cast the tree into term clusters. We

409 selected the term with the best p-value within each cluster as its representative term and display them in a

410 heatmap.

\section{Data Access}

413 The data sets generated for this study are deposited in the Gene Expression Omnibus Repository under 414 accession number GSEXXXX

\section{Acknowledgements}

417 We wish to acknowledge the contribution of Dr Debbie Palmer, Professor Susan Prescott and the Childhood

418 Allergy and Immunology Research team for their contribution to collection of data and samples pertaining 419 to the cohort studied here.

\section{$421 \quad$ Footnotes}

422 Supplemental material is available for this article. 


\section{References}

Bonelli M, Shih H-Y, Hirahara K, Singelton K, Laurence A, Poholek A, Hand T, Mikami Y, Vahedi G, Kanno Y, et al. 2014. Current Topics in Microbiology and Immunology. Curr Top Microbiol 381: 279-326.

Buenrostro JD, Giresi PG, Zaba LC, Chang HY, Greenleaf WJ. 2013. Transposition of native chromatin for fast and sensitive epigenomic profiling of open chromatin, DNA-binding proteins and nucleosome position. Nat Methods 10: 1213-1218.

Champhekar A, Damle SS, Freedman G, Carotta S, Nutt SL, Rothenberg EV. 2015. Regulation of early T-lineage gene expression and developmental progression by the progenitor cell transcription factor PU.1. Gene Dev 29: 832-48.

Chisolm DA, Savic D, Moore AJ, Ballesteros-Tato A, León B, Crossman DK, Murre C, Myers RM, Weinmann AS. 2017. CCCTC-Binding Factor Translates Interleukin 2- and $\alpha-$ Ketoglutarate-Sensitive Metabolic Changes in T Cells into Context-Dependent Gene Programs. Immunity 47: 251-267.e7.

Corces MR, Trevino AE, Hamilton EG, Greenside PG, Sinnott-Armstrong NA, Vesuna S, Satpathy AT, Rubin AJ, Montine KS, Wu B, et al. 2017. An improved ATAC-seq protocol reduces background and enables interrogation of frozen tissues. Nat Methods 14: 959-962.

Gearing LJ, Cumming HE, Chapman R, Finkel AM, Woodhouse IB, Luu K, Gould JA, Forster SC, Hertzog PJ. 2019. CiiiDER: A tool for predicting and analysing transcription factor binding sites. Plos One 14: e0215495.

Heinz S, Romanoski CE, Benner C, Glass CK. 2015. The selection and function of cell type-

Henriksson J, Chen X, Gomes T, Ullah U, Meyer KB, Miragaia R, Duddy G, Pramanik J, Yusa K, Lahesmaa R, et al. 2019. Genome-wide CRISPR Screens in T Helper Cells Reveal

Hnisz D, Abraham BJ, Lee TI, Lau A, Saint-André V, Sigova AA, Hoke HA, Young RA. 2013.

Li P, Spolski R, Liao W, Leonard WJ. 2014. Complex interactions of transcription factors in mediating cytokine biology in T cells. Immunol Rev 261: 141-56.

Martino D, Maksimovic J, Joo J-H, Prescott SL, Saffery R. 2012. Genome-scale profiling reveals a subset of genes regulated by DNA methylation that program somatic T-cell phenotypes in humans. Genes Immun 13: 388-398.

Martino D, Neeland M, Dang T, Cobb J, Ellis J, Barnett A, Tang M, Vuillermin P, Allen K, Saffery R. 2018. Epigenetic dysregulation of naive CD4+ T-cell activation genes in childhood food allergy. Nat Commun 9: 3308 . 
464

Martino DJ, Bosco A, McKenna KL, Hollams E, Mok D, Holt PG, Prescott SL. 2011. T-cell activation genes differentially expressed at birth in CD4+ T-cells from children who develop $\operatorname{IgE}$ food allergy: Microarray gene profiling in children with IgE-mediated food allergy. Allergy 67: 191-200.

Phan AT, Goldrath AW, Glass CK. 2017. Metabolic and Epigenetic Coordination of T Cell and Macrophage Immunity. Immunity 46: 714-729.

Qian F-C, Li X-C, Guo J-C, Zhao J-M, Li Y-Y, Tang Z-D, Zhou L-W, Zhang J, Bai X-F, Jiang Y, et al. 2019. SEanalysis: a web tool for super-enhancer associated regulatory analysis. Nucleic Acids Res 47: W248-W255.

Rawlings JS, Gatzka M, Thomas PG, Ihle JN. 2010. Chromatin condensation via the condensin II complex is required for peripheral T-cell quiescence: Chromatin condensation is required for quiescence. Embo J 30: 263-276.

Ross SH, Cantrell DA. 2018. Signaling and Function of Interleukin-2 in T Lymphocytes. Annu Rev Immunol 36: 411-433.

Scharer CD, Blalock EL, Barwick BG, Haines RR, Wei C, Sanz I, Boss JM. 2016. ATAC-seq on biobanked specimens defines a unique chromatin accessibility structure in naïve SLE B cells. Sci Rep-uk 6: 27030.

Schep AN, Buenrostro JD, Denny SK, Schwartz K, Sherlock G, Greenleaf WJ. 2015. Structured nucleosome fingerprints enable high-resolution mapping of chromatin architecture within regulatory regions. Genome Res 25: 1757-1770.

Schones DE, Cui K, Cuddapah S, Roh T-Y, Barski A, Wang Z, Wei G, Zhao K. 2008. Dynamic Regulation of Nucleosome Positioning in the Human Genome. Cell 132: 887-898.

Simovski B, Vodák D, Gundersen S, Domanska D, Azab A, Holden L, Holden M, Grytten I, Rand K, Drabløs F, et al. 2017. GSuite HyperBrowser: integrative analysis of dataset collections across the genome and epigenome. Gigascience 6: gix032.

Thurman RE, Rynes E, Humbert R, Vierstra J, Maurano MT, Haugen E, Sheffield NC, Stergachis AB, Wang H, Vernot B, et al. 2012. The accessible chromatin landscape of the human genome. Nature 489: 75-82.

Tough DF, Rioja I, Modis LK, Prinjha RK. 2020. Epigenetic Regulation of T Cell Memory: Recalling Therapeutic Implications. Trends Immunol 41: 29-45.

Ungerbäck J, Hosokawa H, Wang X, Strid T, Williams BA, Sigvardsson M, Rothenberg EV. 2018. Pioneering, chromatin remodeling, and epigenetic constraint in early T-cell gene regulation by SPI1 (PU.1). Genome Res 28: 1508-1519.

Wolf T, Jin W, Zoppi G, Vogel IA, Akhmedov M, Bleck CKE, Beltraminelli T, Rieckmann JC, Ramirez NJ, Benevento M, et al. 2020. Dynamics in protein translation sustaining T cell preparedness. Nat Immunol 1-11. 
Yan F, Powell DR, Curtis DJ, Wong NC. 2020. From reads to insight: a hitchhiker's guide to ATAC-seq data analysis. Genome Biol 21: 22.

502 Yue F, Cheng Y, Breschi A, Vierstra J, Wu W, Ryba T, Sandstrom R, Ma Z, Davis C, Pope BD, 503 et al. 2014. A comparative encyclopedia of DNA elements in the mouse genome. Nature 515: $504 \quad 355-64$.

505 Yukawa M, Jagannathan S, Vallabh S, Kartashov AV, Chen X, Weirauch MT, Barski A. 2020. AP-1 activity induced by co-stimulation is required for chromatin opening during T cell activationAP-1 controls T cell chromatin remodeling. J Exp Medicine 217. 2019. Metascape provides a biologist-oriented resource for the analysis of systems-level

510 datasets. Nat Commun 10: 1523. 